\title{
Research of the Model of Traffic Information Acquisition and Distribution Based on VANET
}

\author{
Lan Qiu, Juan Wang \\ College of information engineering, \\ Jiangxi University of Technology, Nanchang, \\ Jiangxi, 330098,China
}

\begin{abstract}
At present, mainstream traffic information acquisition methods cannot directly get the vehicle speed and traffic density, but VANET-based broadcast mode can be very good to meet this requirement. This paper studies the traffic information distribution to collect traffic information based on VANET. In order to reduce the redundant information of the broadcast and restrict broadcast storms, this paper puts forward the traffic information distribution method based on the priority broadcast. In this method, traffic information distribution is limited to specific section.
\end{abstract}

Keywords-VANET;information acquisition; information distribution

\section{INTRODUCTION}

In recent years, intelligent transportation system played a key role on improving traffic problems. As a new form of wireless network, vehicular Ad Hoc network has become a new hot research topic. Its purpose is to build a self-organizing inter-vehicle communication network, which is easy deployment, low cost and open architecture. At present, mainstream traffic information acquisition methods cannot directly get the vehicle speed and traffic density. However, VANETbased traffic information system can collect both traffic flow parameters through the information broadcast. But in the urban scene, there is high density of vehicles and it is easy to produce broadcast redundancy. In this paper, a traffic information distribution method based on the priority broadcast is proposed. In this method, traffic information broadcast is limited to specific section. It can reduce the redundant information of the broadcast and restrict broadcast storms.

\section{THE MODEL OF TRAFFIC INFORMATION ACQUISITION AND DISTRIBUTION}

In the collection of traffic information, broadcast communication has more advantages than unicast communication. So broadcast as a means of communication is inevitable. According to the hop count of the transmission, data broadcasting can be divided into single hop and multi hop broadcast. Single hop broadcast sends information to the neighbor node in the one hop range. Multi hop broadcast realizes the multi hop data transmission.

Many researchers make efforts in order to improve the reliability and performance of broadcast protocols. Such as literature [1] presents a reliable broadcast protocol with high reliability which can provide priority service to different traffic information[1]. Some scholars presents the waiting time of the receiving node to forward the broadcast information by dynamically adjusting in order to adapt to the change of density of nodes, and puts forwards solving network segmentation results in information accessibility issues by reverse neighbor car scratch which can suppress redundant better[2]. There is also a study of accident distribution algorithm aimed at high speed road scene which can reduce the misjudgment and overhead in order to improve the precision[3].

Literature [4] proposed a model of traffic information acquisition and distribution, which is a information acquisition model by multi hop broadcast[4]. As shown in Figure 1, When the vehicle enters the new sections, it records the starting position and time stamp. When the vehicle leaves this section (reach the acquisition terminal),it records the ending position and time stamp. So it can compute the average speed information of the vehicle in this section. Then it will broadcast the driving information , but only broadcast to the vehicle of this section ,so the information broadcast direction and traffic flow will in the opposite direction. 


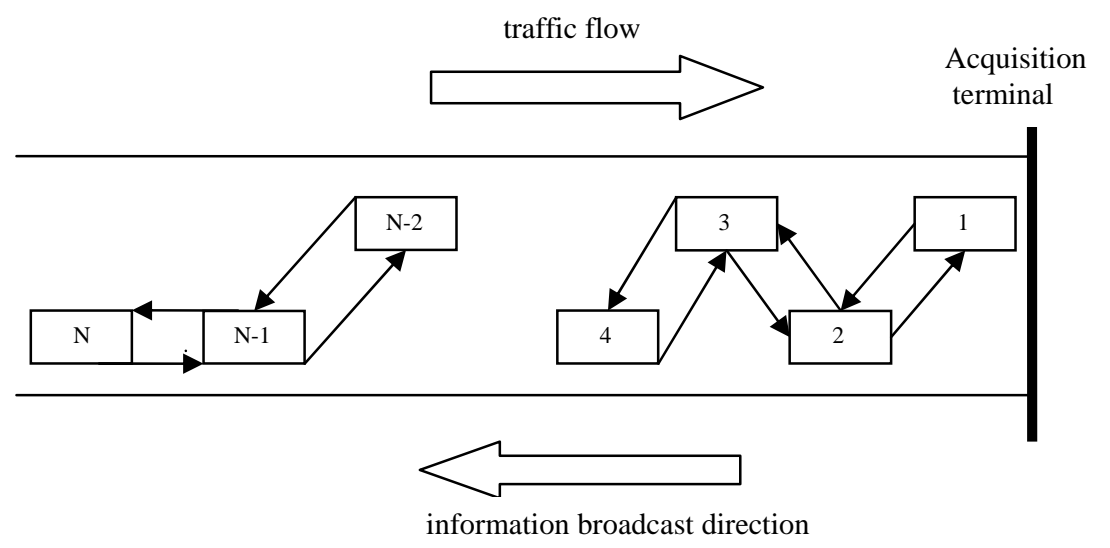

Figure 1. A model of traffic information acquisition and distribution

This communication model will broadcast limit in this section, and only the vehicle of this section can access to the road traffic information. The advantages of this model is that broadcast is triggered only when vehicle arrives acquisition terminal rather than periodic broadcast to reduce the number of broadcast packets and burden of the network.

To limit the broadcast storm, this paper also take the information acquisition method which reversing broadcast in this section as the same as the communication model. But in order to avoid information delay and improve the information value, this paper uses the periodic broadcast mechanism. Then the vehicle which just enters the road can get more road information. But in the flooding broadcast mechanism, the vehicle behind will repeatedly receive information from the vehicle in front. So this paper uses priority index to realize multi hop forwarding of traffic report.

\section{TRAFFIC INFORMATION DISTRIBUTION BASED ON PRIORITY BROADCAST}

\section{A. The basic idea of priority broadcast}

The basic idea of using priority index to realize multi hop forwarding of traffic report is that When the vehicle receives a new traffic report, it will enter the priority competition process, then calculate waiting time according to the priority index. Then, the vehicle enter the waiting time in order to decide whether to forward the traffic report. In the waiting time, if the vehicle has received an identical traffic report ,then the other neighbor vehicle has priority to forward this traffic report, and the vehicle will exit competitive priority program. Otherwise the vehicle will get priority right.

In this paper, the formula for the waiting time:

$$
\text { WTi = Tmax-PI }
$$

In the formula, WTi is the waiting time of the vehicle $i$, Tmax is the maximum waiting time of the vehicle, PI is the priority index. In this paper, using the distance to determine the priority right, so the PI is defined as follows:

$$
\mathrm{PI}=\max [0,(\mathrm{Rr}-\mathrm{Rt}+\mathrm{d}) / \mathrm{Rr}]
$$

In the formula, $\mathrm{Rr}$ is the wireless transmission radius of the receiving node, $\mathrm{Rt}$ is the wireless transmission radius of the emission node, $\mathrm{d}$ is the distance between two nodes. Because $d$ could not be greater than Rt, so the value range of PI is $[0,1]$. When the d closer to Rt, the priority index will higher, and easily get the priority right.

\section{B. Traffic information definition}

At present, traffic detection technology which only uses the vehicle occupancy rate to represent the density of vehicles is difficult to detect the density information of vehicle , and the speed data is also very difficult to detect. But vehicle speed and traffic density can be realized conveniently on VANET. As long as the vehicle broadcasts traffic reports including the information in Table 1.

The vehicle ID can use the plate number to uniquely identify the vehicle information, so this paper uses vehicle IP address as the vehicle ID. In this paper, the two road (the former road and the next road) will be identified a journey, also indicated the direction of travel of the vehicle

TABLE I. TRAFFIC REPORTS INFORMATION

\begin{tabular}{|c|c|c|c|c|}
\hline \multirow{2}{*}{$\begin{array}{l}\text { Vehicle } \\
\text { ID }\end{array}$} & \multicolumn{2}{|c|}{ Road ID } & \multirow{2}{*}{ vehicle } & time \\
\cline { 2 - 3 } & the former & the next & speed & stamp \\
\hline ip_ & prev_rnode & next_rnode & spd_ & ts_ \\
\hline
\end{tabular}

In this paper, the HELLO beacon will be used to implement multi hop traffic information distribution. HELLO beacon is used to obtain the position information of the neighbor vehicles within one hop in routing protocol. This paper use it to realize the real-time collection of traffic information in multi hop broadcast.

struct hdr_uror_hello\{

u_int8_t type_; // information type

double $x_{-} ; \quad / /$ the position information of vehicles

double y_;

int prev_rnode; // the former road

int next_rnode; // the next road

double spd_; // vehicle speed 


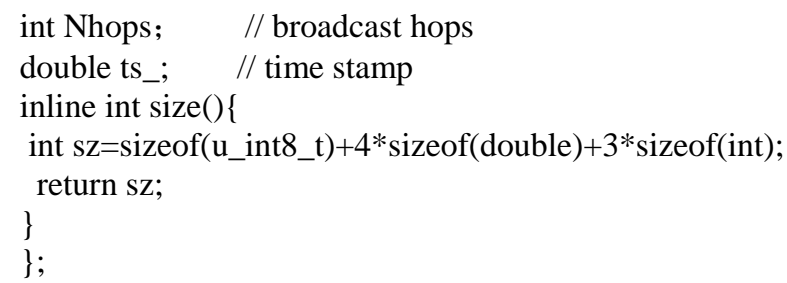

\section{Traffic information distribution based on Priority} broadcast

The competition program process is as Figure 2.The distribution of traffic information is restricted between vehicles traveling in the same sections. The distribution direction is in the opposite direction to the direction of travel. Therefore, from the information amount, the amount of traffic information which is obtained by the vehicle of the starting position is the most. The distribution of traffic information will be achieved by priority competition, the vehicle which competes failure still stores the traffic report.

In order to prevent too many traffic reports, it can arrange the period of each record life for 10 seconds. It will be updated when receive the same vehicle report. In the life period and deleted when Beyond life report. When the vehicle left the road, all the road traffic reports will be deleted.

Vehicle constantly receives the traffic report of the vehicle from the same road, and updates the the traffic report of the same vehicle by the time stamps. When it receives traffic information request message of other vehicles, the new report of each vehicle in this section will be extracted, got together, and the polymerization results will be returned to the requesting vehicles.

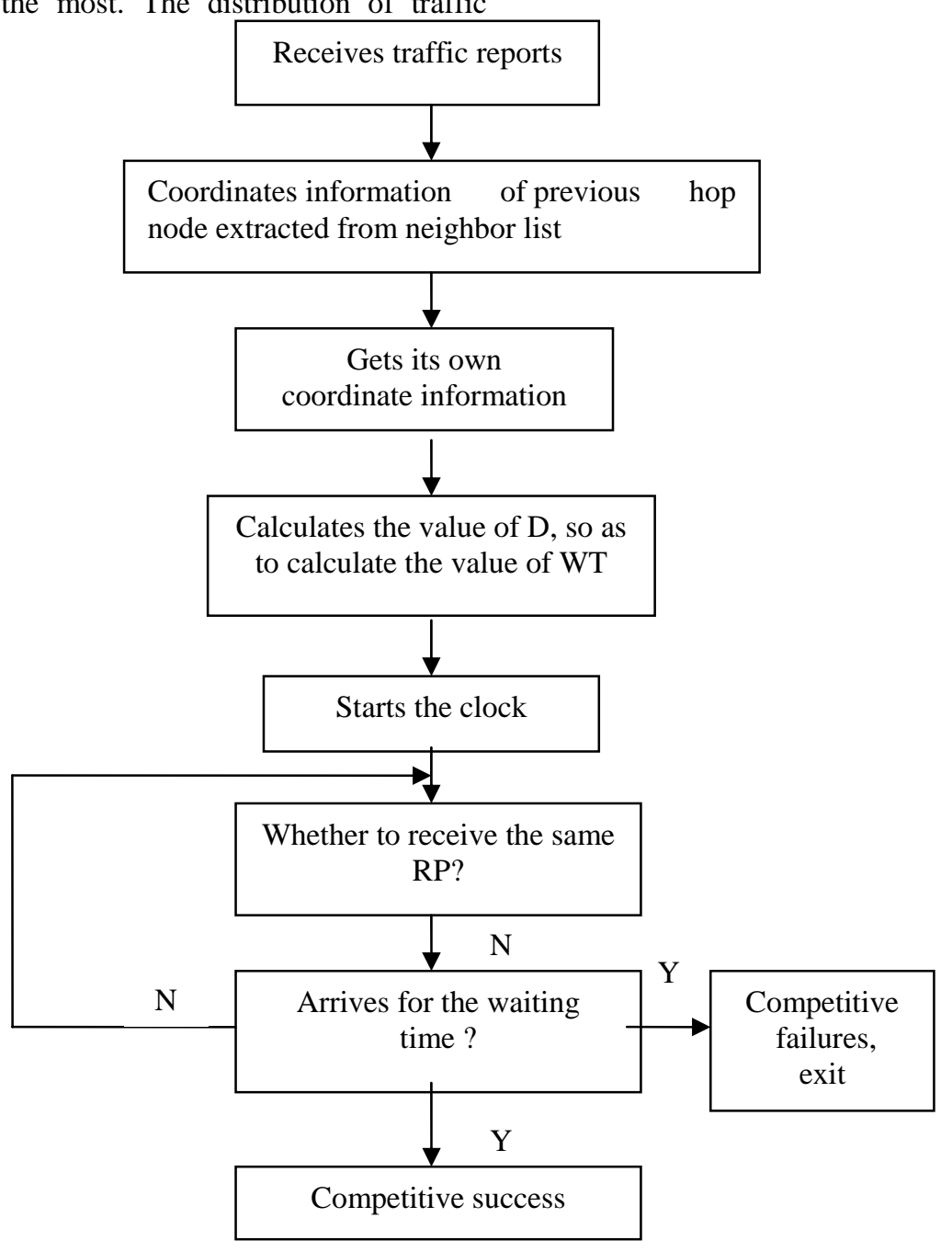

Figure 2. The competition program flow chart 


\section{BROADCAST PERFORMANCE SIMULATION}

In this simulation experiment, the coverage range is $500 \mathrm{~m}$ and each vehicle sends broadcast information. The simulation result is shown in Figure 3. The horizontal axis represents the number of vehicles, the vertical axis represents achievable rate \& rebroadcast saving rate , star data point represents the achievable rate of the flooding broadcast, the circle represents the achievable rate of the priority broadcast, diamond represents rebroadcast saving rate of the priority broadcast.

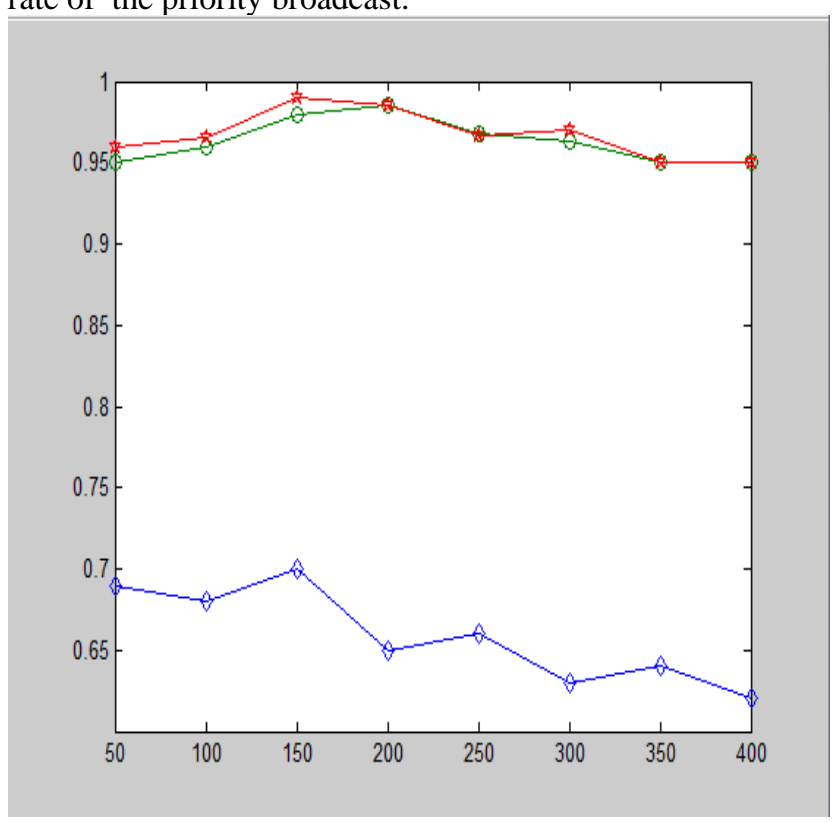

Figure 3. The simulation result

According to the simulation results, with the increasing of the vehicle number, achievable rate is rising then decreasing slowly. This is because when the vehicle is sparse , network connectivity is poor, so the achievable rate is reducing. And when the vehicle is densely, the increase of packet collision will be caused up to reduce the achievable rate. But overall the achievable rate is maintained at a high level (all above 90\%) which is acceptable for this application. In addition, the priority broadcast reaches similar achievable rate with the flooding broadcast, but priority broadcast saves rate more than $60 \%$. So, priority broadcast removes the redundant broadcast of the flooding broadcast, with high efficiency, and has little effect on the achievable rate.

\section{CONCLUSIONS}

This paper studies the traffic information distribution to collect traffic information based on VANET. In order to reduce the redundant information of the broadcast and restrict broadcast storms, this paper puts forward the traffic information distribution method based on the priority broadcast. In this method, traffic information distribution is limited to specific section. This paper uses priority index to realize multi hop forwarding of traffic report.

\section{Acknowledgements}

This paper is one of the achievements of Natural Science Fund Project " Research of VANET real-time traffic information system for the car network " (ZR13QN09) from the Jiangxi Institute of Technology .

\section{REFERENCES}

[1] Balon N,Guo Jinhua.Increasing broadcast reliability in vehicular ad hoc networks[A].Proceedings of the 3rd international workshop on Vehicular ad hoc networks[c].New York:ACM Press ,2006,104-105.

[2] Yu Q Y. Heijenk G.Abiding geocast for warning message dissemination in vehicular ad hoc networks[A].Proceedings of the IEEE International Conference on Communications Workshops[C].New York:IEEE Press,2008,400-404.

[3] Huang Ching-Ling, Guan Xu, FALLAH Y P, et al. Robustness evaluation of decentralized self-information dissemination control algorithms for VANET tracking applications[A].Proceedings of the 70th Vehicular Technology Conference[C].USA :IEEE,2009,1-5.

[4] Baoling Wang, Qi Zhang. Method of traffic information acquisition based on inter vehicle communication [J].Mo dern electronic technology , 2011,34 ( 7 ) : 202-204. 